\title{
Brain Morphometry and Cognitive Performance in Detoxified Alcohol-Dependents with Preserved Psychosocial Functioning
}

\author{
Sandra Chanraud', Catherine Martelli ${ }^{2}$, Francoise Delain ${ }^{2}$, Nikoletta Kostogianni ${ }^{3}$, Gwenaelle Douaud $^{4}$, \\ Henri-Jean Aubin ${ }^{3}$, Michel Reynaud ${ }^{2}$ and Jean-Luc Martinot*,' \\ 'Inserm U.797, CEA-INSERM Research Unit 'Neuroimaging \& Psychiatry', Institute for Health and Medical Research (INSERM) and Atomic \\ Energy Commission (CEA) Hospital Department Frédéric Joliot, IFR49, Univ Paris-sud, Univ Paris 5, Orsay, France; ${ }^{2}$ APHP, Department of \\ Psychiatry and Addictology, Paul Brousse Hospital, Villejuif, France; ${ }^{3}$ APHP, Addiction Treatment Center, Emile Roux Hospital, Limeil-Brevannes, \\ France; ${ }^{4}$ URA CNRS-CEA 2210, Frédéric Joliot Department (SHFJ), Orsay, France
}

\begin{abstract}
The extent of structural brain damage and related cognitive deficits has been little described in alcohol-dependent individuals with preserved social functioning. Thus, we investigated the relationship between regional alterations, executive performance, and drinking history. Volumes of gray and white matter were assessed using magnetic resonance imaging voxel-based morphometry in healthy men and in detoxified alcohol-dependent men with good psychosocial functioning. Their executive performance was assessed using neuropsychological tests. Regression analyses were carried out in the regions in which volume differences were detected. Decreases in gray matter were detected bilaterally in alcohol-dependents in the dorsolateral frontal cortex (up to 20\% lower), and to a lesser extent in the temporal cortex, insula, thalamus, and cerebellum. Decreases in white matter volume were widespread, being up to $10 \%$ in corpus callosum. The degradation of neuropsychological performance correlated with gray matter volume decreases in the frontal lobe, insula, hippocampus, thalami and cerebellum, and with white matter decrease in the brainstem. An early age at first drinking was associated with decreased gray matter volumes in the cerebellum, brainstem (pons), and frontal regions. Regional alteration in gray and white matter volume was associated with impairment of executive function despite preserved social and somatic functioning in detoxified patients. Besides involving frontal regions, these findings are consistent with a cerebello-thalamo-cortical model of impaired executive functions in alcohol-dependent individuals.

Neuropsychopharmacology (2007) 32, 429-438. doi: I0.1038/sj.npp. I30 I219; published online 18 October 2006
\end{abstract}

Keywords: alcoholism; brain; neuropsychology; gray matter; white matter; voxel-based morphometry

\section{INTRODUCTION}

Heavy alcohol consumption can lead to severe psychosocial impairments and affect nearly every organ system (DSM IV TR). Although there is a substantial literature regarding its incidence on structural brain damage (eg Fein et al, 2002; Hommer et al, 2001; Pfefferbaum et al, 1992) and neuropsychological function (Parsons and Nixon, 1998; Oscar-Berman et al, 1997) in detoxified alcohol-dependents, no study has explored its consequences in alcoholdependents with preserved psychosocial functioning.

In subjects with severe alcohol-dependence, computed tomography (CT), and magnetic resonance imaging (MRI) studies have consistently shown reduced gray and white

\footnotetext{
*Correspondence: Dr J-L Martinot, U797 INSERM-CEA, Service Hospitalier Frédéric Joliot, CEA, 4, place du Général Leclerc, 91401 Orsay, France, Tel: + 33 | 6986 7742, Fax: + 33 | 69867810 ,

E-mail: jean-luc.martinot@cea.fr

Received 24 May 2006; revised 28 July 2006; accepted 28 August 2006 Online publication: 8 September 2006 at http://www.acnp.org/ citations/Npp09080606034l/default.pdf
}

matter volumes, with marked losses in the frontal lobes, the medial temporal, and parietal cortices, in subcortical structures (thalamus, caudate, and lenticularis nuclei) and in the cerebellar cortex (Brewer and Perrett, 1971; Jernigan et al, 1991; Pfefferbaum et al, 1992; Shear et al, 1996; Sullivan, 2003a; Sullivan et al, 2003b). Thinning of the corpus callosum (Pfefferbaum et al, 1996), reduced volume in the pons (Sullivan et al, 2003b) and in the cerebellar vermis (Sullivan et al, 2000) were also reported.

Alcohol-dependence has been consistently associated with mild-to-severe neuropsychological impairments (Parsons and Nixon, 1998). The selective alteration of executive functions, such as planning or problem solving, has been reported even in neurologically normal alcoholic patients (Oscar-Berman et al, 1997). The signs of cognitive dysfunction may occur up to 10 years before alcohol-related neurological disorders appear (Tuck and Jackson, 1991), and it has been suggested that chronic alcohol consumption may lead to early effects on cognitive operations that critically rely on frontal lobe function (Adams et al, 1993; Noel et al, 2001a). 
Despite marked decreases in the volume of the frontal regions during long-term severe alcohol-dependence, previous studies failed to show significant relationship between the volume of these regions and executive function deficits (Ratti et al, 1999; Wang et al, 1993). However, these studies focused on particular brain regions, which may explain the lack of relationship found between volumes and neuropsychological deficits.

Nevertheless, relationships between brain function deficits and neuropsychological impairments have been previously reported in neurologically normal alcoholdependent subjects (Adams et al, 1993; Dao-Castellana et al, 1998; Noel et al, 2001a). For example, Dao-Castellana et al (1998) observed in a PET study a significant relationship between the extent of left dorsolateral and medial prefrontal hypometabolism and lower reaction times in the Stroop test and lower verbal fluency performance.

Automated whole-brain methods are valuable techniques for investigating the relationships between brain morphometry and executive functions. There are at least three reasons for this. The first is that automated methods are not limited by observer-dependent factors. Second, this approach allows the volume of the subcortical regions, such as subcortical nuclei or cerebellum to be investigated, as non-frontal gray matter has recently been suggested to contribute to complex cognitive functions (Daum and Ackermann, 1995; Middleton and Strick, 2000; Schmahmann and Pandya, 1997). Also, there have been few studies examining both the volume of these regions and the executive performance (Sullivan, 2003a).

The final reason is that this approach allows the analysis of white matter to be included. It may be that cognitive impairment is also related to differences in the white matter (Pfefferbaum et al, 2000). Only two studies explored the relationships between the volume of white matter and executive performances (Gazdzinski et al, 2005; Sullivan, 2003a) and no study addressed this issue in alcoholdependents preserved in social functioning.

In the present study, we used a whole-brain, voxel-based morphometry (VBM) approach and selected neuropsychological tests that assess executive functions, to (1) search for widespread decreases in gray and white matter volumes, (2) investigate executive functioning, and (3) look for relationships between regional volumes and drinking history and neuropsychological tests scores. We hypothesized that between-group regional volume differences would underlie executive performance impairment in alcohol-dependent group.

\section{SUBJECTS AND METHODS}

We included 31 patients with alcohol-dependence (DSM-IV criteria) and 28 healthy subjects. All were Caucasian, righthanded males. Patients were recruited on admission to detoxification or day-care units in two addiction departments of the Paul Brousse and Emile Roux hospitals in the Paris area (Assistance Publique, Hôpitaux de Paris).

A senior psychiatrist (CM, JLM, and HJA) interviewed and clinically evaluated patients, as well as examined their medical records and biological data. The medical consequences of chronic alcoholism on other organs may confound the association between brain morphometry and behavioral measures. Thus, as brain changes and cognitive deficits are partly secondary to liver damage in alcoholdependent patients with alcoholic cirrhosis (Arria et al, 1991), we selected detoxified drinkers with no clinical evidence of brain dysfunction or medical conditions considered to be clinical indicators of severe alcoholism (eg alcohol-induced dementia or chronic liver disease). Patients were included if they fulfilled the following inclusion criteria: (1) patients must have had less than three periods of withdrawal because more than two periods of withdrawal may be associated with greater cognitive impairment in alcohol-dependent subjects (Duka et al, 2003); (2) patients had to have been detoxified for at least 3 weeks and to be abstaining as assessed by biological norms (normal levels of gamma-glutamyl-transferase (GGT) and normal levels of carbohydrate-deficient transferring (CDT); and (3) they must not have taken lorazepam or sedative medication for at least 7 days.

Exclusion criteria were: (1) signs or symptoms of malnutrition; (2) signs of liver dysfunction, assessed by an aspartate aminotransferase/alanin aminotransferase (AST/ ALT) ratio greater than two (Cohen and Kaplan, 1979); and (3) high scores on Hamilton anxiety and Hamilton depression scales (see Table 1).

We recruited healthy control subjects from the community facilities. Inclusion criteria were: (1) an alcohol consumption less than two equivalent standard alcoholic drinks per week and (2) a score less than or equal to five on the Alcohol Use Disorders Identification Test (AUDIT; Reinert and Allen, 2002).

For both groups, exclusion criteria comprised being under 25 or over 65 years of age, being left-handed, non-

Table I Alcohol-Dependent Subjects' Characteristics $(m \pm S D)$

\begin{tabular}{lc}
\hline Patients' characteristics & Value \\
\hline Hamilton depression & $1.65 \pm 1.35$ \\
Hamilton anxiety & $1.88 \pm 1.39$ \\
& \\
Alcohol consumption & $24.2 \pm 14.4$ \\
Consumption (drinks per day during the 3 months & \\
preceding detoxification ${ }^{\mathrm{a}}$ ) & $8 \pm 6.3$ \\
Duration of dependence (years) & $26.4 \pm 29$ \\
Abstinence (weeks) & $20.5 \pm 6.48$ \\
Age at first drinking & $39 \pm 11.1$ \\
Age at onset of dependence & \\
& \\
Biological variables & $51.96 \pm 6.78(<53)^{\mathrm{b}}$ \\
GGT & $27.85 \pm 13.54(<38)^{\mathrm{b}}$ \\
ALT level (U/I) & $27 \pm 14.32(<40)^{\mathrm{b}}$ \\
AST level (U/I) & $1.07 \pm 0.23$ \\
AST/ALT & $1.97 \pm 0.25(<2.6)^{\mathrm{b}}$ \\
CDT & \\
\hline
\end{tabular}

AST, aspartate aminotransferase; ALT, alanin aminotransferase; GGT, gammaglutamyl-transferase; CDT, carbohydrate-deficient transferring.

a One drink was a beverage containing approximately $10 \mathrm{~g}$ of ethanol (standardization of beer, wine, spirit).

baboratory norms. 
fluent in French, drug abuse (other than nicotine), anxiety or depressive disorders and neurological, somatic or other psychiatric symptoms, including a history of head injury with loss of consciousness, stroke, or other major brain abnormalities observed on MRI scans.

Subjects were assessed on the AUDIT scale and the Social Adjustment Scale Self Report (SAS-SR; Weissman and Bothwell, 1976). The SAS-SR is a self-report questionnaire that evaluates daily functioning, and includes questions on social and leisure activities, relationships with the marital partner, children and extended family, and perception of economic status.

The study was approved by the Bicêtre ethics committee. After the nature and aim of the tests and the MRI scan were explained, written informed consent was obtained from each subject.

\section{Neuropsychological Tests}

Typically, all participants underwent the Mini Mental State Examination (MMSE; Folstein et al, 1975) and a battery of neuropsychological tests either on the day of or up to 3 days after the MRI examination. These neuropsychological tests included the following tests sensitive to frontal dysfunction (Rezai et al, 1993). (1) The Trail Making Test Part B (TMTB; Reitan, 1958). This investigates visual-conceptual and visual-motor tracking skills, concentrating on psychomotor speed, divided attention, mental flexibility, and the ability to shift. The total time to complete the test was taken as the test score. (2) The Wisconsin Card Sorting Test (WCST; Heaton and Pendleton, 1981). This assesses abstract thinking, cognitive flexibility, concept identification, hypothesis generation, and the ability to use response feedback information. Test scores of interest were the number of categories sorted and the number of persevering responses. (3) The Letter Fluency Test (LFT; Cardebat et al, 1990). This assesses verbal fluency and the total number of correct words produced was taken as the test score. (4) The Stroop Color Word test (Treisman and Fearnley, 1969). This concentrates on selective attention, perceptual interference, and information processing speed. An interference score was derived by subtracting the prediction of performance on the third task (based on the performance on the first two tasks) from the actual performance on the third task. (5) The Letter-Number Sequencing test (Wechsler, 1981). This assesses 'working memory' that is, the ability to simultaneously recall and organize similar or different types of stimuli. The examiner presents combinations of two to nine letters and numbers and asks subjects to repeat the numbers in ascending order and then the letters in alphabetical order (eg the correct response for 9-L-2-A would be $2-9-\mathrm{A}-\mathrm{L}$ ). The number of reproduced digits was taken as the test score under the two conditions.

\section{Brain Imaging}

Image acquisition. All subjects underwent volumetric MRI brain scanning using a $1.5 \mathrm{~T}$ Signa imager (General Electric Healthcare, Milwaukee, WI) with a standard 3D T1weighted inversion recovery fast-spoiled gradient-recalled sequence with the following parameters: axial orientation, matrix $=256 \times 192$ interpolated to $256 \times 256,124$ slice locations, $0.9375 \times 0.9375 \mathrm{~mm}^{2}$ in-plane resolution, slice thickness $=1.3 \mathrm{~mm}, \mathrm{TE}=2 \mathrm{~ms}, \mathrm{TR}=10 \mathrm{~ms}, \mathrm{TI}=600 \mathrm{~ms}$, flip angle $=10^{\circ}$, and read bandwidth $=12.5 \mathrm{kHz}$. Owing to motion artifacts and other technical difficulties, only 26 patients and 24 healthy subjects were retained for MRI data analysis (Table 2).

Image preprocessing. All MRI data were processed using SPM2 software (Wellcome Department of Cognitive Neurology, London) running on MATLAB version 7 (The MathWorks, Natick, MA).

MRI images were analyzed using the optimized approach of VBM developed by Good et al (2001). This is a fully automated whole-brain technique that provides a voxelwise assessment of regional gray and white cerebral matter. VBM analysis includes following steps: First, study-specific templates of gray and white matter were created for automated segmentation and spatial normalization of the initial images. These templates were created from the images of alcohol-dependent patients and healthy subjects to ensure the data from both groups were treated equally during spatial normalization. All images were registered to the 'average 152' template of MNI (Montreal Neurological Institute) using an affine transformation (Ashburner and Friston, 2000), and then segmented into gray matter, white matter, and cerebrospinal fluid (CSF) compartments. Only the gray and white matter images were retained for subsequent processing. Gray and white matter images were then averaged and smoothed using an isotropic $8 \mathrm{~mm}$ FWHM (full width at half maximum) Gaussian smoothing function.

Subsequent image processing included the following steps: First, the native MRI scans were segmented into gray and white matter images then nonlinearly transformed with the corresponding study-specific template to derive the normalization parameters subsequently applied to the initial images. This step allowed optimal spatial normalization of gray and white matter to the customized gray and white matter templates and reduced the contribution of any non-brain voxels. Second, these native images were then resampled with third-order B-spline interpolation to a final voxel size of $1.5 \times 1.5 \times 1.5 \mathrm{~mm}^{3}$. Third, the normalized native images were segmented into gray matter, white matter, and CSF compartments. Fourth, a Jacobian modulation was applied by multiplying the voxel intensities by the Jacobian determinants derived from the nonlinear component of the spatial normalization step. This step allowed to compensate the voxel volume modification induced by nonlinear spatial normalization (Good et al, 2001). Finally, the modulated images from alcohol-dependent and healthy subjects were smoothed with an $8 \mathrm{~mm}$-FWHM isotropic Gaussian smoothing function. After smoothing, each voxel represents the local average amount of gray matter in the surrounding region, the size of which is determined by the smoothing kernel. Smoothing was carried out at $8 \mathrm{~mm}$ FWHM in order to explore volumetric differences as well in cortical than in smaller subcortical regions.

\section{Statistical Analyses}

We tested the normality of the distribution of neuropsychological data using SPSS 8.0 software (SPSS Inc., Chicago, IL) 
Table 2 Means and Standard Deviations $( \pm S D)$ of Demographic and Performance Variables for the Alcohol-Dependent $(n=26)$ and Healthy $(n=24)$ Subjects

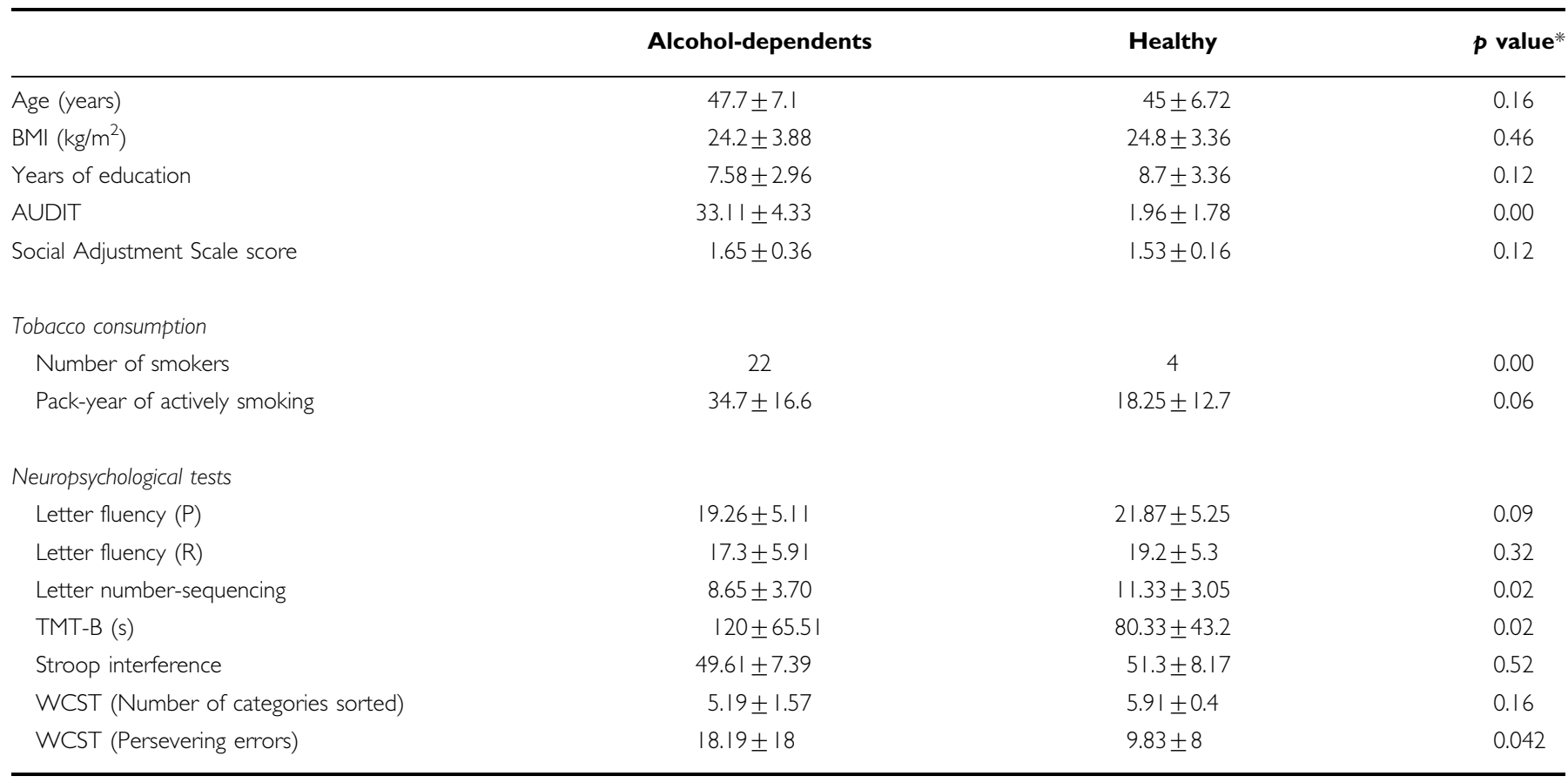

BMI, body mass index; AUDIT, Alcohol Use Disorders Identification Test; TMT-B, Trail Making Test Part-B; WCST, Wisconsin Card Sorting Test. * $p$ values for between-group comparisons with Mann-Whitney U-tests.

and then converted the raw data to $z$ scores by adjusting for age and years of education. As the data was non-normally distributed, we used non-parametric statistical tests. Statistical significance of the between-group differences was calculated by the non-parametric rank-based MannWhitney $U$-test $(p<0.05)$. A Spearman correlation analysis was used to examine the relationships between alcohol consumption variables and cognitive performance, with $p<0.05$ taken as the statistical threshold.

Between-group analysis. Anatomical data were analyzed using the SPM2 software package (http://www.fil.ion.ucl.ac. $\mathrm{uk} / \mathrm{spm} /$ ). Between-group comparisons of gray and white matter were carried out on a voxel basis using the general linear model, based on random Gaussian field theory (Friston, 1995). Differences in overall gray matter and white matter between groups were accounted for by entering age, years of education, and tobacco consumption variables as confounding covariates into the design matrix of the ANCOVA. The height threshold for comparison analyses was set at $p<5 \times 10^{-3}$ FDR-corrected (false discovery rate) for gray and white matter with a spatial extent of 50 voxels. This FDR-corrected threshold ensures that on average no more than $5 \%$ voxels are misclassified as false positives among those voxels in the gray and white matter in which the null hypothesis is rejected (Genovese et al, 2002). Significant peak voxels were reported in MNI template coordinates. Their regional location was obtained by overlaying the $t$-maps on the study-specific template and using the AAL (Tzourio-Mazoyer et al, 2002) and Brodmann atlases accompanying the MRIcro software package (Rorden and Brett, 2000). In addition, percentage of reduction was calculated in regions where strong between-group differences were detected. These percentages of reduction were calculated with SPSS 8.0 software from data of gray/ white matter volumes in regions-of-interest extracted using the Marsbar toolbox (marsbar.sourceforge.net/) implemented in SPM2.

Regression analyses. We examined the relationships between anatomical data and alcohol consumption by regression analyses of the whole-brain imaging data of the alcohol-dependent group with subjects' age entered as a confounding variable. Complementary simple regression analyses between the anatomical data and neuropsychological test scores were carried out within the alcoholic group. Thus, linear regression analyses were carried out in each voxel that had been highlighted by the between-group analysis. SPM $t$-maps from the between-group comparison were used as a mask (Schmitz et al, 2006), and only those scores from neuropsychological tests showing significantly altered performances were entered in the regression analyses. For regression analyses, an exploratory uncorrected statistical threshold was set at $p<0.001$ with a minimal cluster size of 50 voxels. Furthermore, regression analyses between the anatomical data and neuropsychological scores were also carried out within the healthy control group with the same statistical threshold.

\section{RESULTS}

The drinking history variables of the alcohol-dependent group are presented in Table 1. The mean age at first drinking occurred around early adulthood, whereas the reported mean age at onset of dependence was around 40 
Table 3 Regions of Significant Decreases in Gray and White Matter in Alcohol-Dependent Subjects Compared to Healthy Subjects

\begin{tabular}{|c|c|c|c|c|c|c|c|}
\hline \multirow[b]{2}{*}{ Regional clusters } & \multirow[b]{2}{*}{ Side } & \multirow{2}{*}{$\begin{array}{l}\text { Brodmann's } \\
\text { area }\end{array}$} & \multirow{2}{*}{$\begin{array}{l}\text { Cluster size: } \\
\text { voxel number* }\end{array}$} & \multicolumn{3}{|c|}{$\begin{array}{l}\text { MNI coordinates of peak } \\
\text { voxel }\end{array}$} & \multirow[b]{2}{*}{ Maximal Z-value } \\
\hline & & & & $x$ & $y$ & $\mathbf{z}$ & \\
\hline \multicolumn{8}{|l|}{ Gray matter } \\
\hline Dorsolateral frontal \& parietal & L & 6 & 17880 & -44 & -7 & 54 & 7.56 \\
\hline Dorsolateral frontal \& parietal & $\mathrm{R}$ & 6 & 16494 & 40 & -1 & 54 & 6.36 \\
\hline Insula & L & - & 625 & -42 & -9 & -2 & 4.25 \\
\hline Superior temporal & L & 22 & 349 & -64 & -22 & 4 & 4.63 \\
\hline Superior temporal & L & 48 & 625 & -39 & -28 & 10 & 4.25 \\
\hline Thalamus & L & - & 24 & -9 & -3 & 10 & 4.25 \\
\hline Thalamus & $\mathrm{R}$ & - & 153 & 8 & -3 & 10 & 4.38 \\
\hline \multicolumn{8}{|l|}{ White matter } \\
\hline Anterior cingulate, Bilateral anterior frontal & $\mathrm{R}$ & - & 19121 & 4 & 5 & -4 & 5.14 \\
\hline Corpus callosum & - & - & - & 2 & 10 & 21 & 5.07 \\
\hline Anterior paracingulate & L & - & 760 & -15 & 39 & 11 & 4.51 \\
\hline Frontal lobe (subprecentral gyrus) & L & - & 134 & -48 & ।1 & 9 & 4.65 \\
\hline Frontal lobe (submiddle frontal gyrus) & L & - & 55 & -40 & 33 & -6 & 3.81 \\
\hline Frontal lobe (subprecentral gyrus) & L & - & 71 & -43 & -7 & 47 & 3.95 \\
\hline Temporal (subgyral) & L & - & 139 & -35 & -50 & -1 & 3.80 \\
\hline Temporal lobe (submiddle temporal gyrus) & $\mathrm{R}$ & - & 494 & 48 & -3 & -20 & 4.23 \\
\hline Brainstem (pons) & L & - & 161 & -8 & -28 & -31 & 3.97 \\
\hline
\end{tabular}

R, Right; L, left; MNI, Montreal Neurological Institute; Regional clusters of voxels are presented by decreasing $Z$ value.

*Voxel size: $x=1.5, y=1.5, z=1.5 \mathrm{~mm}$.

years of age. Patients had been treated for a chronic intoxication with decreasing doses of sedative medication (lorazepam) and B vitamins (B6 and B1). All patients had a fixed address. Most were active smokers. The groups were similar for mean age, body mass index, years of education and social functioning. The two groups did not differ for MMSE scores (see Table 2).

Neuropsychological performance was impaired in alcohol-dependent subjects for the TMT-B, the WAIS-letter number sequencing, and WCST tests, but was unaffected for the LFT and Stroop interference tests (Table 2). We found no co-variation between any neuropsychological score and age at onset of alcohol-dependence, daily alcohol consumption, and duration of dependence or of abstinence, tobacco use, depression scores, or any biological variable (CDT, GGT, AST, ALT, and AST/ALT).

Between-group comparisons of the neuroimaging data showed significant clusters of decreased gray matter volume bilaterally in dorsolateral frontal cortex extending to the anterior part of the parietal lobe, in the temporal and lingual cortices, in the cingulate and insula, and in subcortical regions such as the thalamus and the cerebellum (crus) (see Table 3). The volume of the dorsolateral frontal cortex decreased by up to $20 \%$ in patients with respect to healthy subjects (Figure 1). Between-group comparisons of white matter showed significant reduction in the corpus callosum, frontal, cingulate, temporal and cerebellar regions, and in the pons (Table 3 ). The maximum decrease was $10 \%$ in the corpus callosum. We found no region in which the gray or white matter volume was higher in alcohol-dependent group than in the healthy group.

Regression analysis of the different regions with alcoholconsumption variables revealed a positive relationship between age at first drinking and gray matter decreases in the right middle frontal gyrus ( $x=27, y=42, z=-8$, cluster size $=127$ voxels, $Z=3.83$ ), the brainstem (basal pons $x=2$, $y=-24, z=-40$, cluster size $=544$ voxels, $Z=3.64)$ and bilaterally in the cerebellum (crus $x=-32, y=-70, z=$ -50 , cluster size $=507$ voxels, $Z=3.81 ; x=39, y=-75$, $z=-50$ cluster size $=415$ voxels, $Z=3.60$ ) (Figure 2). No other alcohol-consumption variable co-varied with regional differences in volume of gray or white matter.

Exploratory regional regression analysis between neuropsychological scores and gray matter volumes in alcoholdependent patients revealed a positive correlation between the WAIS-letter number sequencing scores and gray matter 
a

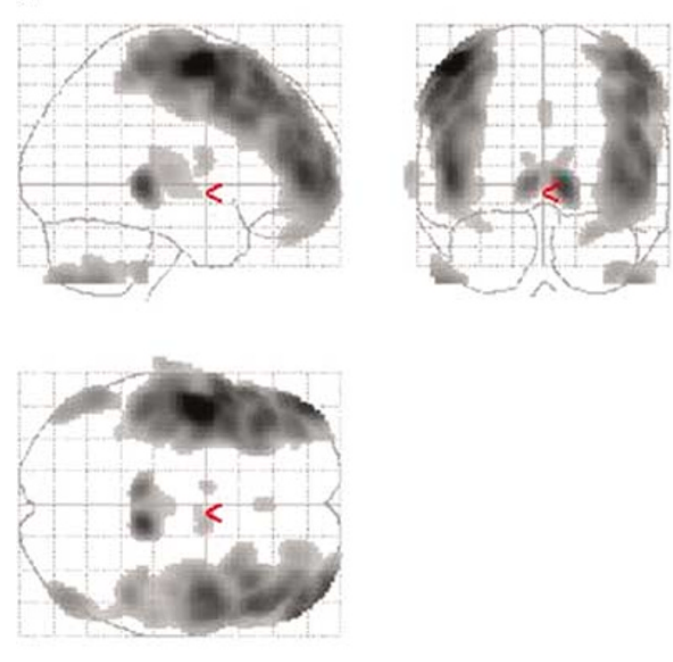

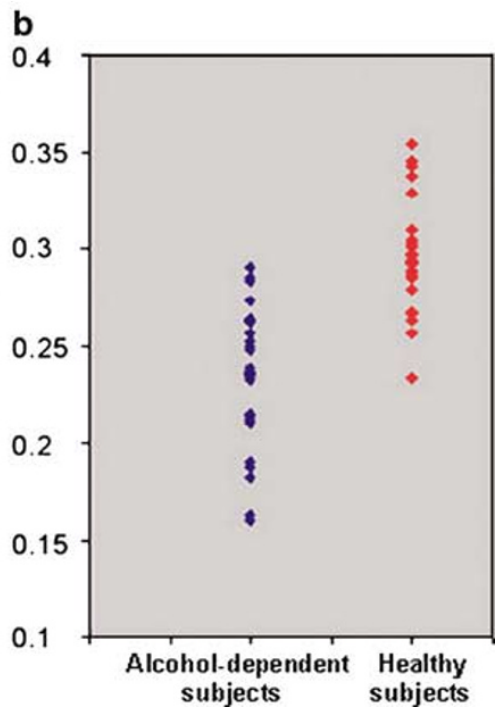

Figure I (a) Gray matter volume regional decreases in alcohol-dependents as compared to healthy subjects using SPM2 software. (b) Individual plotted and normalized voxel values, in alcohol-dependent group on the left and healthy group on the right, at the highest peak-voxel detected by the betweengroup comparison (MNI coordinates: $x=-44, y=-7, z=54$ ).
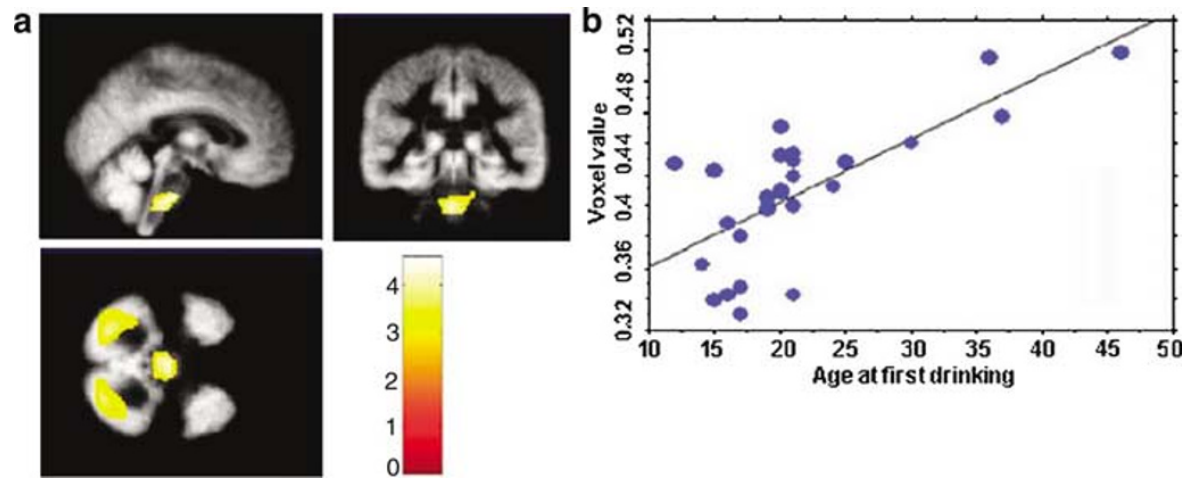

Figure 2 (a) Regions where positive correlation between gray matter volume and age at first drinking was detected in alcohol-dependent subjects are rendered on a template image. The colored bar represents the $t$-score of significant voxels. (b) Plot at MNI coordinates in cerebellum $(x=-32, y=-70$ $z=-50$ ) showing the variation of voxel value with the age (years) at first drinking. Regression slope: $Y=0.3\left|9+0.004 \times X ; R^{2}=0.5\right| 5$, where $X=$ age at first dinking, and $Y=$ volume of gray matter.

volume bilaterally in the cerebellum $(x=-48, y=-60, z=$ $-48,235$ voxels, $Z=3.77 ; x=50, y=-60, z=-50,164$ voxels, $Z=3.64$ ).

Regression analysis with TMT-B scores revealed extensive clusters in the right superior frontal $(x=22, y=66, z=$ $-15,129$ voxels, $Z=3.58)$, right superior temporal $(x=63$, $y=-27, z=4,170$ voxels, $Z=3.77$ ), precentral (bilaterally) $(x=62, y=10, z=32,127$ voxels, $Z=3.90 ; x=-33, y=4$, $z=60,61$ voxels, $Z=3.35)$ and postcentral $(x=-68, y=$ $-22, z=22,104$ voxels, $Z=3.64$ ), as well as in the insula $(x=42, y=4, z=-6,79$ voxels, $Z=3.29)$ and the left hippocampus ( $x=-12, y=-38, z=2,61$ voxels, $Z=3.20$ ).

Regression analysis with WCST persevering errors score revealed a significant positive relationship with gray matter volume in the middle temporal gyri (bilaterally) $(x=45$, $y=-64, z=3,698$ voxels, $Z=3.05 ; x=-42, y=-66, z=8$, 201 voxels, $Z=2.87)$, the parahippocampus $(x=-18, y=$ $-26, z=-15,196$ voxels, $Z=2.85$ ), the thalamus (bilaterally) ( $x=-4, y=-15, z=3,292$ voxels, $Z=2.84 ; x=-2$, $y=-18, z=8,292$ voxels, $Z=2.76$ ), and the cerebellum $(x=20, y=-30, z=-20,1041$ voxels, $Z=3.30)$.

Regional regression analysis between the neuropsychological scores and white matter volumes in alcohol-dependent patients revealed a positive correlation between performance on the WAIS-letter number sequencing test and white matter volumes in the left temporal cortex $(x=54$, $y=8, z=-24,285$ voxels, $Z=3.14$ ) and bilaterally in the brainstem $(x=-16, y=-28, z=-28,230$ voxels, $Z=3.51$; $x=16, y=-26, z=-26,237$ voxels, $Z=3.44$ ). TMT-B performance positively correlated with white matter volumes bilaterally in the supramarginal region $(x=60, y=-32$, $z=27,285$ voxels, $Z=3.40 ; x=-56, y=-42, z=26,331$ voxels, $Z=3.29)$, the corpus callosum $(x=0, y=27, z=-2$, 109 voxels, $Z=3.32$ ) and bilaterally in the brainstem $(x=12, y=-3, z=-18,306$ voxels, $Z=3.75 ; x=-18$, $y=-21, z=-21,75$ voxels, $Z=3.49$ ).

We found no relationship between the volumes of the different regions of white matter and WCST performance. 
Finally, no significant relationship was found within the healthy control group between the regional volumes and neuropsychological scores.

\section{DISCUSSION}

We investigated differences in brain morphometry and the relationship between regional volume and neuropsychological impairment in detoxified alcohol-dependent subjects. The patients had no neurological, somatic or psychiatric complications and the onset of dependence occurred late in life. Furthermore, these patients showed no social function impairment as assessed by the SAS-SR, and were all in employment. This sample involved mainly Type A alcoholics as designated by Babor et al (1992).

Neuropsychological performances revealed impaired executive functions in alcohol dependents. Also, the neuropsychological scores of the patients correlated with gray matter volumes in the frontal and temporal cortices, the insula, the hippocampus, the thalami, and the cerebellum, as well as with white matter volumes in the brainstem (pons and midbrain). No similar relationship were found between brain imaging and neuropsychological data in healthy control group. These findings suggest that brain damage and neuropsychological impairment exist, and are interrelated in abstaining alcohol-dependent patients with preserved social functioning. Furthermore, this pattern of correlations is characteristic of this alcohol-dependent group. The age at first drinking significantly correlated with the decrease in gray matter volume in the frontal cortex, the cerebellum and the brainstem. No other drinking history variables were significantly related to decreases in gray or white matter volumes.

The neuropsychological performance of the alcoholdependent participants showed that most executive functions were impaired, except for the Stroop and verbal fluency tasks. Although these two tasks are sensitive to frontal lobe damage, this pattern of impairment is consistent with previous studies of alcohol-dependent subjects (Noel et al, 2001b; Ratti et al, 2002).

Some studies have suggested that cognitive performance worsens in direct proportion to the severity and duration of alcoholism (Beatty et al, 2000; Parsons and Nixon, 1998). However, as in other studies, we detected no relationship between neuropsychological performance and drinking history or alcohol-related biological variables (Noel et al, 2001b; Schafer et al, 1991).

The alterations in the volume of gray matter in the different brain regions of the patient in our study are consistent with those identified in previous neuroimaging studies using a regions-of-interest approach. They are also consistent with documented neuropathological abnormalities in neurologically normal alcoholics. Indeed, both macroscopic and histological abnormalities have been reported separately in fronto-parietal cortices, the cerebellum and subcortical regions (hypothalamus and thalamus) (Harper and Matsumoto, 2005). In our study, we showed significant decreases in gray matter volume in all these brain regions using a whole-brain VBM approach and thus our approach was not limited only to analyzing particular regions.

We observed a maximum decrease in gray matter volume in the dorsolateral frontal cortex, reaching up to $20 \%$ reduc- tion. Although the variable measured with VBM is different from neuron counting, the magnitude of the gray matter decrease in this region is consistent with results using histological approaches (Kril and Harper, 1989). Our findings further support the 'accelerated ageing' hypothesis that suggests that the changes in the brains of alcoholdependent individuals mimic those found in older non-alcohol-dependent subjects. Consistent with this hypothesis, we observed structural changes in a sample of subjects with a mean age under 50 years in same areas as than those observed in elderly healthy subjects (Noonberg et al, 1985). These results are most striking when considering that the alcohol-dependent subjects remained socially functional.

For alteration to white matter, abstaining alcoholdependent subjects had smaller white matter volumes than healthy subjects, notably in the frontal and temporal lobes, in cingulate, corpus callosum, pons, and in the cerebellum. The magnitude of the white matter difference in the corpus callosum (10\%) was similar to that reported by Pfefferbaum et al (1996) and is consistent with most neuropathological studies (de la Monte, 1988; Harper and Kril, 1985; Kril et al, 1997; O'Neill et al, 2001). Our results of widespread white matter deficits also agree with a recent report showing widespread alteration in diffusion tensor imaging (Pfefferbaum et al (2006) and suggest that alcohol has a direct neurotoxic effect.

Age at first drinking was the only drinking history variable that correlated with a decrease in gray matter volume in the middle frontal cortex, brainstem (pons), and cerebellum. This suggests that these regions are more vulnerable to early-onset drinking, despite dependence occurring later. As the decrease in prefrontal cortex and in cerebellum size has also been observed in adolescents with alcohol-dependence, these regions may be the first to be affected by alcohol (De Bellis et al, 2005). Furthermore, age at first drinking seems to play a crucial role in later substance dependence, as early alcohol use has been associated with abuse and dependence on licit and illicit substances later in life (Grant et al, 2006). Thus, our results further add to the suggestion that the neurotoxic effects of alcohol may impede the maturation of these brain regions in adolescents. Nevertheless, we found no correlation between brain volumes and anamnestic or biological variables assessing alcohol exposure. Neither the duration, amount of alcohol intake, nor any biological variables co-varied with regional volumes. This suggests that a combination of rather than a single variable may be associated with alterations of brain morphometry. Further, longitudinal studies are needed to assess the impact of these alcohol-related variables.

For the relationship between brain volume and executive function, reductions in gray matter volume were primarily located in the frontoparietal regions and reductions in white matter volume were widespread, whereas we found that neuropsychological performance was related gray matter volume in several brain regions but only in one region for white matter region: the brainstem.

Impairment in TMT-B score correlated with decreases in gray matter volume in the frontal and temporal cortices, the insula, and the hippocampus. This test is considered to require the use of various higher-order cognitive functions 
and distributed networks. The TMT-B test is associated with cognitive demands on 'set shifting', 'cognitive flexibility' and a 'general attention component' (eg Zakzanis et al, 2005). fMRI in healthy subjects showed that TMT-B engages the left frontal and temporal lobes, the cingulate cortex and the insula (Zakzanis et al, 2005). Our results provide further evidence that the cognitive processes underlying the TMT-B performance occur in these brain regions.

Moreover, we observed that WCST scores of alcoholdependent patients co-varied with the gray matter volumes in the middle temporal gyri, thalamus, and cerebellum, but were not related to decreases in frontal gray matter volume. A lack of correlation between the morphometry of the prefrontal gray matter and WCST scores has been previously reported in alcohol-dependent subjects (Sullivan, 2003a). Although the WCST is used as an executive test, these results suggest that brain shrinkage elsewhere from the frontal cortex may play a role in alterations in highorder planning performances. Our findings also suggest the subcortical nuclei play a critical role, as the volume of the thalamus co-varied with the WCST scores. fMRI studies in healthy subjects have shown that the thalamus underlies 'set shifting' during negative feedback (Monchi et al, 2001), a process that required during the WCST (Alexander et al, 1986).

The working memory impairment of our patient sample, as assessed by the WAIS letter-number sequencing task, was correlated with decreases in cerebellum volume, which is consistent with this structure being involved in executive functioning (see Schmahmann and Pandya, 1997 for review).

Although executive deficits in alcohol-dependent subjects co-varied with gray matter volume decreases in a widely distributed set of regions, WAIS letter-number sequencing and TMT-B performance was associated particularly with volume decrease in one white matter region, the brainstem. The brainstem includes large white matter bundles (ie the superior cerebellar and cerebral peduncles). Thus, white matter alterations of the brainstem may contribute to executive impairment.

Classically, executive performance impairment relates to frontal alterations (eg Smith and Jonides, 1999). However, many brain region volumes that related to executive performances in the present patient sample involved nonfrontal regions forming part of the cerebello-thalamocortical circuit, reported to underlie, and modulate cognitive functions (Middleton and Strick, 2000; Schmahmann and Pandya, 1997). Without ruling out frontal involvement, our findings are consistent with the theory of Sullivan (Sullivan, 2003a) in which the impairment of cognitive performance in alcohol-dependent subjects may be based on primary or modulatory influences from subcortical and brainstem structures. Our results further extend this hypothesis and suggest that subcortical shrinkage within cerebello-thalamocortical circuits may adversely affect frontal functioning. Although these regions may not affect perceptual processes, they would have a bottleneck effect on central decisionmaking processes (Sigman and Dehaene, 2005).

The results reported here should be considered in light of certain limitations. First, the alcohol consumption data were collected retrospectively, and thus may sensitive to the subjectivity inherent in the anamnestic method. However, we detected no association between brain volume and alcohol consumption estimates, either with anamnestic variables or with objective alcohol-related biological variables. Second, the regression analyses of cross-sectional data do not allow for causal conclusions. Thus, any causal interpretation between regional brain shrinkage and poor neuropsychological performance should be dismissed. Furthermore, because this study is cross-sectional, we used the term 'decrease' in order to denote differences in brain tissue volume but here this term does not express a loss of volume overtime (Rohlfing et al, 2006). Third, although the patients were studied after a long detoxification period, the present study was not designed to examine the duration of the changes in morphometry and executive performance. Therefore, we can say nothing regarding the putative irreversibility of the abnormalities. Finally, some limitations are inherent to the VBM approach, in particular to the spatial normalization step (Bookstein, 2001).

In conclusion, the present study has shown evidence of dorsolateral frontal reductions in brain volume and alterations of the cerebello-thalamo-cortical pathways in clinically and socially preserved alcohol-dependent individuals. We also detected a close relationship between decreases in regional brain matter volumes and neuropsychological impairments. Further longitudinal studies of brain function are needed to determine whether these morphometric alterations initiate degradation of executive functions.

\section{ACKNOWLEDGEMENTS}

The study was supported by grants from the French society of alcohol studies (Société Française d'Alcoologie, SFA) and the Interministry Mission against Drug and Addiction (Mission Interministérielle de Lutte contre la Drogue et la Toxicomanie, MILDT). Each author has no involvement, financial or otherwise, that might potentially bias their work.

We thank the healthy subjects and abstaining alcoholic subjects; Arnaud Cachia, Damien Ringuenet, and Sebastien Meriaux for their help in data analyses and Michele Wessa, Sylvie Berthoz, Christian Trichard, Alexis Roche, and Eric Artiges for thoughtful comments on the manuscript.

\section{REFERENCES}

Adams KM, Gilman S, Koeppe RA, Kluin KJ, Brunberg JA, Dede D et al (1993). Neuropsychological deficits are correlated with frontal hypometabolism in positron emission tomography studies of older alcoholic patients. Alcohol Clin Exp Res 17: 205-210.

Alexander GE, DeLong MR, Strick PL (1986). Parallel organization of functionally segregated circuits linking basal ganglia and cortex. Annu Rev Neurosci 9: 357-381.

Arria AM, Tarter RE, Starzl TE, Van Thiel DH (1991). Improvement in cognitive functioning of alcoholics following orthotopic liver transplantation. Alcohol Clin Exp Res 15: 956-962.

Ashburner J, Friston KJ (2000). Voxel-based morphometry-the methods. Neuroimage 11: 805-821.

Babor TF, Hofmann M, DelBoca FK, Hesselbrock V, Meyer RE, Dolinsky ZS et al (1992). Types of alcoholics, I. Evidence for an empirically derived typology based on indicators of vulnerability and severity. Arch Gen Psychiatry 49: 599-608.

Beatty WW, Tivis R, Stott HD, Nixon SJ, Parsons OA (2000). Neuropsychological deficits in sober alcoholics: influences of 
chronicity and recent alcohol consumption. Alcohol Clin Exp Res 24: 149-154.

Bookstein FL (2001). 'Voxel-based morphometry' should not be used with imperfectly registered images. Neuroimage 14: 1454-1462.

Brewer C, Perrett L (1971). Brain damage due to alcohol consumption: an air-encephalographic, psychometric and electroencephalographic study. $\mathrm{Br} J$ Addict Alcohol Other Drugs 66: $170-182$.

Cardebat D, Doyon B, Puel M, Goulet P, Joanette Y (1990). Formal and semantic lexical evocation in normal subjects. Performance and dynamics of production as a function of sex, age and educational level. Acta Neurol Belg 90: 207-217.

Cohen JA, Kaplan MM (1979). The SGOT/SGPT ratio-an indicator of alcoholic liver disease. Dig Dis Sci 24: 835-838.

Dao-Castellana MH, Samson Y, Legault F, Martinot JL, Aubin HJ, Crouzel C et al (1998). Frontal dysfunction in neurologically normal chronic alcoholic subjects: metabolic and neuropsychological findings. Psychol Med 28: 1039-1048.

Daum I, Ackermann H (1995). Cerebellar contributions to cognition. Behav Brain Res 67: 201-210.

De Bellis MD, Narasimhan A, Thatcher DL, Keshavan MS, Soloff P, Clark DB (2005). Prefrontal cortex, thalamus, and cerebellar volumes in adolescents and young adults with adolescent-onset alcohol use disorders and comorbid mental disorders. Alcohol Clin Exp Res 29: 1590-1600.

de la Monte SM (1988). Disproportionate atrophy of cerebral white matter in chronic alcoholics. Arch Neurol 45: 990-992.

Duka T, Townshend JM, Collier K, Stephens DN (2003). Impairment in cognitive functions after multiple detoxifications in alcoholic inpatients. Alcohol Clin Exp Res 27: 1563-1572.

Fein G, Di Sclafani V, Cardenas VA, Goldmann H, Tolou-Shams M, Meyerhoff DJ (2002). Cortical gray matter loss in treatmentnaive alcohol dependent individuals. Alcohol Clin Exp Res 26: 558-564.

Folstein MF, Folstein SE, McHugh PR (1975). 'Mini-mental state'. A practical method for grading the cognitive state of patients for the clinician. J Psychiatr Res 12: 189-198.

Friston KJ (1995). Statistical parametric mapping in functional imaging: a general linear approach. Hum Brain Mapp 2: 189-210.

Gazdzinski S, Durazzo TC, Studholme C, Song E, Banys P, Meyerhoff DJ (2005). Quantitative brain MRI in alcohol dependence: preliminary evidence for effects of concurrent chronic cigarette smoking on regional brain volumes. Alcohol Clin Exp Res 29: 1484-1495.

Genovese CR, Lazar NA, Nichols T (2002). Thresholding of statistical maps in functional neuroimaging using the false discovery rate. Neuroimage 15: 870-878.

Good CD, Johnsrude IS, Ashburner J, Henson RN, Friston KJ, Frackowiak RS (2001). A voxel-based morphometric study of ageing in 465 normal adult human brains. Neuroimage 14: 21-36.

Grant JD, Scherrer JF, Lynskey MT, Lyons MJ, Eisen SA, Tsuang MT et al (2006). Adolescent alcohol use is a risk factor for adult alcohol and drug dependence: evidence from a twin design. Psychol Med 36: 109-118.

Harper C, Kril J (1985). Brain atrophy in chronic alcoholic patients: a quantitative pathological study. J Neurol Neurosurg Psychiatry 48: 211-217.

Harper C, Matsumoto I (2005). Ethanol and brain damage. Curr Opin Pharmacol 5: 73-78.

Heaton RK, Pendleton MG (1981). Use of neuropsychological tests to predict adult patients' everyday functioning. J Consult Clin Psychol 49: 807-821.

Hommer D, Momenan R, Kaiser E, Rawlings R (2001). Evidence for a gender-related effect of alcoholism on brain volumes. $A m \mathrm{~J}$ Psychiatry 158: 198-204.
Jernigan TL, Butters N, DiTraglia G, Schafer K, Smith T, Irwin M et al (1991). Reduced cerebral grey matter observed in alcoholics using magnetic resonance imaging. Alcohol Clin Exp Res 15: 418-427.

Kril JJ, Halliday GM, Svoboda MD, Cartwright H (1997). The cerebral cortex is damaged in chronic alcoholics. Neuroscience 79: 983-998.

Kril JJ, Harper CG (1989). Neuronal counts from four cortical regions of alcoholic brains. Acta Neuropathol (Berlin) 79: 200-204.

Middleton FA, Strick PL (2000). Basal ganglia and cerebellar loops: motor and cognitive circuits. Brain Res Brain Res Rev 31: 236-250.

Monchi O, Petrides M, Petre V, Worsley K, Dagher A (2001). Wisconsin Card Sorting revisited: distinct neural circuits participating in different stages of the task identified by eventrelated functional magnetic resonance imaging. J Neurosci 21: 7733-7741.

Noel X, Paternot J, Van der Linden M, Sferrazza R, Verhas M, Hanak C et al (2001a). Correlation between inhibition, working memory and delimited frontal area blood flow measure by 99mTc-Bicisate SPECT in alcohol-dependent patients. Alcohol Alcohol 36: 556-563.

Noel X, Van der Linden M, Schmidt N, Sferrazza R, Hanak C, Le Bon $\mathrm{O}$ et al (2001b). Supervisory attentional system in nonamnesic alcoholic men. Arch Gen Psychiatry 58: $1152-1158$.

Noonberg A, Goldstein G, Page HA (1985). Premature aging in male alcoholics: 'accelerated aging' or 'increased vulnerability'? Alcohol Clin Exp Res 9: 334-338.

O'Neill J, Cardenas VA, Meyerhoff DJ (2001). Effects of abstinence on the brain: quantitative magnetic resonance imaging and magnetic resonance spectroscopic imaging in chronic alcohol abuse. Alcohol Clin Exp Res 25: 1673-1682.

Oscar-Berman M, Shagrin B, Evert DL, Epstein C (1997). Impairments of brain and behavior: the neurological effects of alcohol. Alcohol Health Res World 21: 65-75.

Parsons OA, Nixon SJ (1998). Cognitive functioning in sober social drinkers: a review of the research since 1986. I Stud Alcohol 59: 180-190.

Pfefferbaum A, Adalsteinsson E, Sullivan EV (2006). Supratentorial profile of white matter microstructural integrity in recovering alcoholic men and women. Biol Psychiatry 59: 364-372.

Pfefferbaum A, Lim KO, Desmond JE, Sullivan EV (1996). Thinning of the corpus callosum in older alcoholic men: a magnetic resonance imaging study. Alcohol Clin Exp Res 20: $752-757$.

Pfefferbaum A, Lim KO, Zipursky RB, Mathalon DH, Rosenbloom MJ, Lane B et al (1992). Brain gray and white matter volume loss accelerates with aging in chronic alcoholics: a quantitative MRI study. Alcohol Clin Exp Res 16: 1078-1089.

Pfefferbaum A, Sullivan EV, Hedehus M, Adalsteinsson E, Lim KO, Moseley M (2000). In vivo detection and functional correlates of white matter microstructural disruption in chronic alcoholism. Alcohol Clin Exp Res 24: 1214-1221.

Ratti MT, Bo P, Giardini A, Soragna D (2002). Chronic alcoholism and the frontal lobe: which executive functions are imparied? Acta Neurol Scand 105: 276-281.

Ratti MT, Soragna D, Sibilla L, Giardini A, Albergati A, Savoldi F et al (1999). Cognitive impairment and cerebral atrophy in 'heavy drinkers'. Prog Neuropsychopharmacol Biol Psychiatry 23: 243-258.

Reinert DF, Allen JP (2002). The Alcohol Use Disorders Identification Test (AUDIT): a review of recent research. Alcohol Clin Exp Res 26: 272-279.

Reitan RM (1958). Validity of the Trail Making Test as an indicator of organic brain damage. Percept Mot Skills 8: 271-276. 
Rezai K, Andreasen NC, Alliger R, Cohen G, Swayze II V, O’Leary DS (1993). The neuropsychology of the prefrontal cortex. Arch Neurol 50: 636-642.

Rohlfing T, Sullivan EV, Pfefferbaum A (2006). Deformationbased brain morphometry to track the course of alcoholism: differences between intra-subject and inter-subject analysis. Psychiatry Res 146: 157-170.

Rorden C, Brett M (2000). Stereotaxic display of brain lesions. Behav Neurol 12: 191-200.

Schafer K, Butters N, Smith T, Irwin M, Brown S, Hanger P et al (1991). Cognitive performance of alcoholics: a longitudinal evaluation of the role of drinking history, depression, liver function, nutrition, and family history. Alcohol Clin Exp Res 15: 653-660.

Schmahmann JD, Pandya DN (1997). The cerebrocerebellar system. Int Rev Neurobiol 41: 31-60.

Schmitz N, Rubia K, Daly E, Smith A, Williams S, Murphy DG (2006). Neural correlates of executive function in autistic spectrum disorders. Biol Psychiatry 59: 7-16.

Shear PK, Sullivan EV, Lane B, Pfefferbaum A (1996). Mammillary body and cerebellar shrinkage in chronic alcoholics with and without amnesia. Alcohol Clin Exp Res 20: 1489-1495.

Sigman M, Dehaene S (2005). Parsing a cognitive task: a characterization of the mind's bottleneck. PLoS Biol 3: e37.

Smith EE, Jonides J (1999). Storage and executive processes in the frontal lobes. Science 12: 1657-1661.

Sullivan EV (2003a). Compromised pontocerebellar and cerebellothalamocortical systems: speculations on their contributions to cognitive and motor impairment in nonamnesic alcoholism. Alcohol Clin Exp Res 27: 1409-1419.
Sullivan EV, Deshmukh A, Desmond JE, Lim KO, Pfefferbaum A (2000). Cerebellar volume decline in normal aging, alcoholism, and Korsakoff's syndrome: relation to ataxia. Neuropsychology 14: 341-352.

Sullivan EV, Rosenbloom MJ, Serventi KL, Deshmukh A, Pfefferbaum A (2003b). Effects of alcohol dependence comorbidity and antipsychotic medication on volumes of the thalamus and pons in schizophrenia. Am J Psychiatry 160: $1110-1116$.

Treisman A, Fearnley S (1969). The Stroop test: selective attention to colours and words. Nature 222: 437-439.

Tuck RR, Jackson M (1991). Social, neurological and cognitive disorders in alcoholics. Med J Aust 155: 225-229.

Tzourio-Mazoyer N, Landeau B, Papathanassiou D, Crivello F, Etrad O, Delcroix N et al (2002). Automated anatomical labeling of activations in SPM using a macroscopic anatomical parcellation of the MNI MRI single-subject brain. Neuroimage 15: 273-289.

Wang GJ, Volkow ND, Roque CT, Cestaro VL, Hitzemann RJ, Cantos EL et al (1993). Functional importance of ventricular enlargement and cortical atrophy in healthy subjects and alcoholics as assessed with PET, MR imaging, and neuropsychologic testing. Radiology 186: 59-65.

Wechsler D (1981). Manual for the Wechsler Adult Intelligence Scale-Revised (WAIS-R). The Psychological Corporation: San Antonio, TX.

Weissman MM, Bothwell S (1976). Assessment of social adjustment by patient self-report. Arch Gen Psychiatry 33: 1111-1115.

Zakzanis KK, Mraz R, Graham SJ (2005). An fMRI study of the Trail Making Test. Neuropsychologia 43: 1878-1886. 